\title{
An effective absorbing boundary condition for linear long-wave and linear dispersive-wave tsunami simulations
}

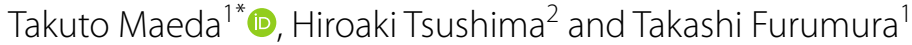

\begin{abstract}
We numerically simulated the propagation of tsunami waves with finite difference methods by using perfectly matched layer (PML) boundary conditions to effectively eliminate artificial reflections from model boundaries. The PML method damps the tsunami height and velocity of seawater only in directions perpendicular to the boundary. Although the additional terms required to implement the PML conditions make the use of the PML technique difficult for linear dispersive tsunami waves, we have proposed an empirical extension of the PML method for modeling dispersive tsunami waves. Even for heterogeneous, realistic bathymetries, numerical tests demonstrated that the PML boundary condition dramatically decreased artificial reflections from model boundaries compared to the use of traditional boundary conditions. The use of PML boundary conditions for numerical modeling of tsunamis is especially useful because it facilitates use of the later phases of tsunamis that would otherwise be compromised by artifacts caused by reflections from model boundaries.
\end{abstract}

Keywords: Tsunami wave, Numerical simulation, Linear long-wave, Dispersive tsunami wave, Finite difference method, Absorbing boundary condition, Perfectly matched layer (PML)

\section{Background}

Tsunami simulation for a near-field earthquake is usually performed with a bounded-area model having a spatial extent on the order of 100-1000 km (e.g., Tanioka 2006; Furumura et al. 2011) rather than with a whole-earth model in the case of a far-field tsunami. At the offshore side of the model boundary, an open or an absorbing condition is necessary to avoid artificial reflections from the physical model boundaries. Simulations by finite difference methods applied to the long-wave tsunami equation usually utilize a one-way wave equation or the Sommerfeld radiation condition (e.g., Hwang et al. 1972; Blumberg and Kantha 1985); alternatively, a sponge condition, which artificially attenuates the wave height in the vicinity of the model boundary, also has been widely used (e.g., Cerjan et al. 1985; Furumura and Saito 2009). These

\footnotetext{
*Correspondence: maeda@eri.u-tokyo.ac.jp

1 Earthquake Research Institute, The University of Tokyo, 1-1-1 Yayoi, Bunkyo-ku, Tokyo 113-0032, Japan

Full list of author information is available at the end of the article
}

absorbers generally work to reduce artificial tsunami reflections from the outer of the model; however, some remaining reflection noises contaminate the later phase in the tsunami waveform. To make full use of the later or full tsunami waveform, therefore the area of the simulation should be enlarged to postpone the arrival of artificial reflections.

Recent development of high-resolution observations at the ocean-bottom cabled instruments (e.g., González and Kulikov 1993) and numerical simulations (e.g., Saito et al. 2010) enabled us to utilize dispersive tsunami waves including later phase. The dispersive tsunami simulation is now being used for inversion studies (e.g., Hossen et al. 2015a; Saito et al. 2011) as well as forward modeling (e.g., Baba et al. 2015; Grilli et al. 2013). In the dispersive tsunami simulation, the existing Sommerfeld radiation condition (e.g., Tanioka 1999) and its extension for weakly dispersive tsunami wave (Kim et al. 1988), or the sponge boundary condition (e.g., Saito et al. 2010) is used so that the same problem of the bounded tsunami simulation arises to utilize later phases or full tsunami waveforms. 
Since the computational load for the dispersive tsunami simulation is quite heavy particularly due to their implicit scheme calculation requirement and a considerable number of iteration to solve governing equation of the dispersive tsunami, the development of more effective absorbing boundary is desirable for dispersive tsunami simulation and also for non-dispersive tsunami simulations to make full use of whole tsunami waveform with keeping the simulation area small and to reduce computational load.

In this report, we propose the use of an alternative and more effective boundary condition based on the perfectly matched layer (PML) method for finite difference numerical modeling of tsunami propagation. The PML method was originally proposed for numerical simulations of electromagnetic waves (Berenger 1994) and is known to be one of the most effective absorbing boundary conditions for wave-related equations. The PML method uses an absorbing region surrounding the model area. Within the full wavefield, only variables with a component of movement perpendicular to the model boundary are attenuated to avoiding artificial reflection. Waves moving parallel to the model boundary are unchanged.

The PML boundary condition has previously been applied successfully to elastic wave propagation (Chew and Liu 1996). This application was followed by many successful applications in earthquake seismology (e.g., Marcinkovich and Olsen 2003; Festa and Nielsen 2003; Maeda and Furumura 2013). The PML method has also been successfully applied to general shallow water equations (e.g., Navon et al. 2004; Lavelle and Thacker 2008) in climate simulation and ocean modeling. In the present study, we focused on application of this technique to the problem of modeling the propagation of tsunami waves, including dispersive tsunami waves.

In the following report, we first show that the PML boundary condition is easily implemented in the case of linear long-wave tsunami equations. Although it is difficult to directly apply the PML boundary condition to a more realistic linear, dispersive-wave tsunami model, we propose an empirical extension of the PML boundary condition to the linear, dispersive tsunami equation. We then demonstrate the effectiveness of the PML absorbing boundary condition under simple and realistic bathymetric conditions.

\section{Perfectly matched layer and its application to tsunami equations}

Linear long-wave tsunami equation with PML

The propagation of a tsunami is governed by the following continuity and momentum equations under the linear long-wave (LLW) assumption (e.g., Goto 1984):

$$
\begin{aligned}
& \frac{\partial \eta}{\partial t}=-\frac{\partial M}{\partial x}-\frac{\partial N}{\partial y} \\
& \frac{\partial M}{\partial t}=-g h \frac{\partial \eta}{\partial x}, \quad \frac{\partial N}{\partial t}=-g h \frac{\partial \eta}{\partial y},
\end{aligned}
$$

where $\eta$ is the tsunami height measured from the undisturbed sea surface, $M$ and $N$ are the horizontal components of the tsunami velocity vertically integrated from the bottom of the ocean to the undisturbed sea surface (hereafter referred to as the tsunami velocity) in the $x$ and $y$-directions, respectively.

The PML regions used to damp outgoing tsunami waves were placed surrounding the numerical model with a finite thickness (usually 10-20 grids) separating them from the interior region. In this PML region, physical variables were decomposed into directions according to the directions of their $x$ and $y$ derivatives. For Eq. 1, the continuity equation, we split the tsunami height $\eta$ into $x$ and $y$-directions as follows:

$$
\begin{aligned}
& \eta=\eta_{(x)}+\eta_{(y)} \\
& \frac{\partial \eta_{(x)}}{\partial t}=-\frac{\partial M}{\partial x}, \quad \frac{\partial \eta_{(y)}}{\partial t}=-\frac{\partial N}{\partial y} .
\end{aligned}
$$

Coordinate stretching in the complex plane was then applied in the frequency domain to attain absorption along a specific direction. We adopted stretching on the complex plane as follows (e.g., Moczo et al. 2007):

$\frac{\partial}{\partial x} \rightarrow \frac{1}{\beta_{(x)}+\delta_{(x)} /(-i \omega)} \frac{\partial}{\partial x}, \quad \frac{\partial}{\partial y} \rightarrow \frac{1}{\beta_{(y)}+\delta_{(y)} /(-i \omega)} \frac{\partial}{\partial y}$,

where $\left(\beta_{(x)}, \beta_{(y)}\right)$ and $\left(\delta_{(x)}, \delta_{(y)}\right)$ are attenuation functions along the $x$ - and $y$-directions, respectively. By applying Eq. 5 to Eq. 4 in the frequency domain, we obtained PML equations as follows:

$$
\begin{aligned}
& -i \omega \beta_{(x)} \eta_{(x)}+\delta_{(x)} \eta_{(x)}=-\frac{\partial M}{\partial x} \\
& -i \omega \beta_{(y)} \eta_{(y)}+\delta_{(y)} \eta_{(y)}=-\frac{\partial N}{\partial y} .
\end{aligned}
$$

When converted to the time domain, the above equations become

$$
\begin{aligned}
& \beta_{(x)} \frac{\partial \eta_{(x)}}{\partial t}+\delta_{(x)} \eta_{(x)}=-\frac{\partial M}{\partial x} \\
& \beta_{(y)} \frac{\partial \eta_{(y)}}{\partial t}+\delta_{(y)} \eta_{(y)}=-\frac{\partial N}{\partial y} .
\end{aligned}
$$


If $\delta_{(x)}$ and $\delta_{(y)}$ are positive, the tsunami height is expected to decrease exponentially with time. The PML equations for tsunami momentum, Eq. 2, were derived in the same manner as follows:

$$
\begin{aligned}
& \beta_{(x)} \frac{\partial M}{\partial t}+\delta_{(x)} M=-g h \frac{\partial \eta}{\partial x} \\
& \beta_{(y)} \frac{\partial N}{\partial t}+\delta_{(y)} N=-g h \frac{\partial \eta}{\partial y},
\end{aligned}
$$

where we note that no further decomposition is required.

In the PML approach, only a wave propagating perpendicular to the model boundary is absorbed so as to avoid artificial reflection from the boundary. A wave propagating parallel to the boundary should be unchanged (Chew and Liu 1996). In a direction parallel to the boundary, we therefore set the attenuation functions $\beta_{(x)}=1$ and $\delta_{(x)}=0$, or $\beta_{(y)}=1$ and $\delta_{(y)}=0$ in Eqs. 7 and 8. Inside the PML region, we followed Zhang and Shen (2010) by adopting a second-order polynomial scaling function to gradually decrease the attenuation functions:

$$
\delta_{(x)}=\delta_{0}\left(\frac{x}{L}\right)^{2}, \quad \beta_{(x)}=1+\left(\beta_{0}-1\right)\left(\frac{x}{L}\right)^{2},
$$

where $L$ is the thickness of the PML region and $x$ is the distance from the beginning of the PML region measured from the boundary between the PML and interior regions. The coefficients $\delta_{0}$ and $\beta_{0}$ are the maximum values of $\delta_{(x)}$ and $\beta_{(x)}$. They were chosen in accordance with the specifications in Zhang and Shen (2010). Note that we used spatially varying $\beta_{(x)}$ value (e.g., Moczo et al. 2007; Maeda and Furumura 2013). In the interior region, except for the PML, the LLW tsunami equations, Eqs. 1 and 2, were solved directly.

\section{Extension to a linear dispersive-wave tsunami model}

To simulate a tsunami propagating in the deep ocean and/or a tsunami with a shorter wavelength, it is necessary to use a linear dispersive-wave (LDW) tsunami model to take into consideration tsunami dispersion effects (e.g., Saito and Furumura 2009). To simulate a dispersive tsunami, we adopted a Boussinesq-type twodimensional LDW equation with vertically integrated tsunami velocities (e.g., Saito et al. 2010) as follows:

$$
\begin{aligned}
& \frac{\partial M}{\partial t}=-g h \frac{\partial \eta}{\partial x}+\frac{1}{3} h^{2} \frac{\partial^{2}}{\partial x \partial t}\left(\frac{\partial M}{\partial x}+\frac{\partial N}{\partial y}\right) \\
& \frac{\partial N}{\partial t}=-g h \frac{\partial \eta}{\partial y}+\frac{1}{3} h^{2} \frac{\partial^{2}}{\partial y \partial t}\left(\frac{\partial M}{\partial x}+\frac{\partial N}{\partial y}\right) .
\end{aligned}
$$

We obtained the tsunami height by using Eq. 1, the continuity equation. The tsunami dispersion effect is caused by the last term on the right-hand side of Eq. 10. It is expected that the dispersion strengthens with increasing ocean depth, $h$.
In the LDW tsunami model, the stretching method cannot be applied straightforwardly to Eq. 10 because there are higher-order derivatives along the mixed directions in the second terms. To cope with this difficulty, we here propose an approximate two-step scheme for empirically incorporating dispersion effects into the PML equation. First, we evaluate the effect of dispersion in the LDW equation by estimating the dispersion term, $\Phi(x, y$, $t$ ), which is defined as follows:

$$
\begin{aligned}
\Phi(x, y, t) & \equiv \frac{1}{3} h^{2} \frac{\partial}{\partial t}\left(\frac{\partial M}{\partial x}+\frac{\partial N}{\partial y}\right) \\
\frac{\partial M}{\partial t} & =-g h \frac{\partial \eta}{\partial x}+\frac{\partial \Phi}{\partial x} \\
\frac{\partial N}{\partial t} & =-g h \frac{\partial \eta}{\partial y}+\frac{\partial \Phi}{\partial y} .
\end{aligned}
$$

After estimating $\Phi$, we evaluated the coordinatestretched PML equation with a weighting factor $w$ as follows:

$$
\begin{aligned}
& \beta_{(x)} \frac{\partial M}{\partial t}+\delta_{(x)} M=-g h \frac{\partial \eta}{\partial x}+w \frac{\partial \Phi}{\partial x} \\
& \beta_{(y)} \frac{\partial N}{\partial t}+\delta_{(y)} N=-g h \frac{\partial \eta}{\partial y}+w \frac{\partial \Phi}{\partial y} .
\end{aligned}
$$

The weighting factor $w$ was chosen so that it smoothly decreased from 1 to 0 in the PML region.

Rigorously speaking, Eq. 11 is equivalent to Eq. 10 only if the bathymetry is flat $(\partial h / \partial x=0, \partial h / \partial y=0)$. We thus assumed a flat bathymetry inside the PML region along the direction perpendicular to the boundary. On the one hand, although Eq. 12 does not exactly adhere to the condition of a PML because the spatial derivatives inside the dispersion term are not stretched, Eq. 12 asymptotes to the PML Eq. 8 as the weighting factor $w$ decreases. On the other hand, the interior domain and the PML region are smoothly connected if $w=1$ on the interface. In that case, the effect of tsunami dispersion is gradually decreased (i.e., Eq. 12 gradually approaches the LLW equation Eq. 8) as the tsunami wave penetrates toward the PML region. We equated the weighting factor $w$ to the following cosine function:

$$
w=\cos \left(\frac{\pi x}{2 L}\right),
$$

where $x$ and $L$ are the distance from the PML-interior interface and the thickness of the PML region, respectively.

\section{Numerical implementation} Linear long-wave tsunami equation

The LLW tsunami equations were numerically solved by using a staggered-grid, finite difference method. The 
spatial coordinates $x$ and $y$ were discretized by spatial grid widths $\Delta x$ and $\Delta y$ defined by $x=i \Delta x\left(i=1, \ldots, N_{x}\right)$, $y=j \Delta y\left(j=1, \ldots, N_{y}\right)$, and time was discretized as $t=n \Delta t\left(n=1, \ldots, N_{t}\right)$. We adopted a staggered-grid layout in accordance with Saito and Furumura (2009), as shown in Fig. 1.

Because the time-marching equations for the PML region, Eqs. 7 and 8, are implicit in time, we used a firstorder Crank-Nicolson scheme (e.g., Press et al. 1986) to solve them numerically. For example, the first equation in Eq. 7 was discretized by using a central finite difference, second-order scheme in both space and time as follows:

$$
\begin{aligned}
& \beta_{(x) i} \frac{\eta_{(x) i, j}^{n+1}-\eta_{(x) i, j}^{n}}{\Delta t}+\delta_{(x) i} \frac{\eta_{(x) i, j}^{n+1}+\eta_{(x) i, j}^{n}}{2} \\
&=-\left(\frac{M_{i+1 / 2, j}^{n+1 / 2}-M_{i-1 / 2, j}^{n+1 / 2}}{\Delta x}\right) .
\end{aligned}
$$

We note that time averaging was applied in the second term on the left-hand side of Eq. 14. By applying this discretization to Eqs. 7 and 8 in the same manner, we obtained the discretized time-marching LLW equations with a PML as follows: and

$$
\begin{aligned}
M_{i-1 / 2, j}^{n+1 / 2}= & \frac{\beta_{(x) i-1 / 2}-\delta_{(x) i-1 / 2} \Delta t / 2}{\beta_{(x) i-1 / 2}+\delta_{(x) i-1 / 2} \Delta t / 2} M_{i-1 / 2, j}^{n-1 / 2} \\
& -\frac{1}{\beta_{(x) i-1 / 2}+\delta_{(x) i-1 / 2} \Delta t / 2} g \bar{h}_{i-1 / 2, j} \frac{\eta_{i, j}^{n}-\eta_{i-1, j}^{n}}{\Delta x} \Delta t \\
N_{i, j-1 / 2}^{n+1 / 2}= & \frac{\beta_{(y) j-1 / 2}-\delta_{(y) j-1 / 2} \Delta t / 2}{\beta_{(y) j-1 / 2}+\delta_{(y) j-1 / 2} \Delta t / 2} N_{i, j+1 / 2}^{n-1 / 2} \\
& -\frac{1}{\beta_{(y) j-1 / 2}+\delta_{(y) j-1 / 2} \Delta t / 2} g \bar{h}_{i, j-1 / 2} \frac{\eta_{i, j}^{n}-\eta_{i, j-1}^{n}}{\Delta y} \Delta t,
\end{aligned}
$$

where $\bar{h}_{i-1 / 2, j} \equiv\left(h_{i, j}+h_{i-1, j}\right) / 2$ and $\bar{h}_{i, j-1 / 2} \equiv\left(h_{i, j}+\right.$ $\left.h_{i, j-1}\right) / 2$ are the averages of the ocean depths along the $x$ - and $y$-directions, respectively. In the PML region, the bathymetry is assumed to be flat along the direction perpendicular to the boundary to reduce reflection. For example, at the $x$-side boundary we set $h_{i, j}=h_{N_{P}, j}$ (for $\left.i=1, \ldots, N_{P}-1\right)$, where $N_{P}$ is the thickness of the PML region in terms of the number of grids. At the model corners, we used homogeneous corner, for example $h_{i, j}=h_{N_{P}, N_{P}}\left(\right.$ for $\quad i=1, \ldots, N_{P}-1$, and $\left.j=1, \ldots, N_{P}-1\right)$. The bathymetries at the other corners are set in the same manner.

$$
\begin{aligned}
\eta_{(x) i, j}^{n+1} & =\frac{\beta_{(x) i}-\delta_{(x) i} \Delta t / 2}{\beta_{(x) i}+\delta_{(x) i} \Delta t / 2} \eta_{(x) i, j}^{n}-\frac{1}{\beta_{(x) i}+\delta_{(x) i} \Delta t / 2}\left(\frac{M_{i+1 / 2, j}^{n+1 / 2}-M_{i-1 / 2, j}^{n+1 / 2}}{\Delta x}\right) \Delta t \\
\eta_{(y) i, j}^{n+1} & =\frac{\beta_{(y) j}-\delta_{(y) j} \Delta t / 2}{\beta_{(y) j}+\delta_{(y) j} \Delta t / 2} \eta_{(y) i, j}^{n}-\frac{1}{\beta_{(y) j}+\delta_{(y) j} \Delta t / 2}\left(\frac{N_{i, j+1 / 2}^{n+1 / 2}-N_{i, j-1 / 2}^{n+1 / 2}}{\Delta y}\right) \Delta t \\
\eta_{i, j}^{n+1} & =\eta_{(x) i, j}^{n+1}+\eta_{(y) i, j}^{n+1}
\end{aligned}
$$

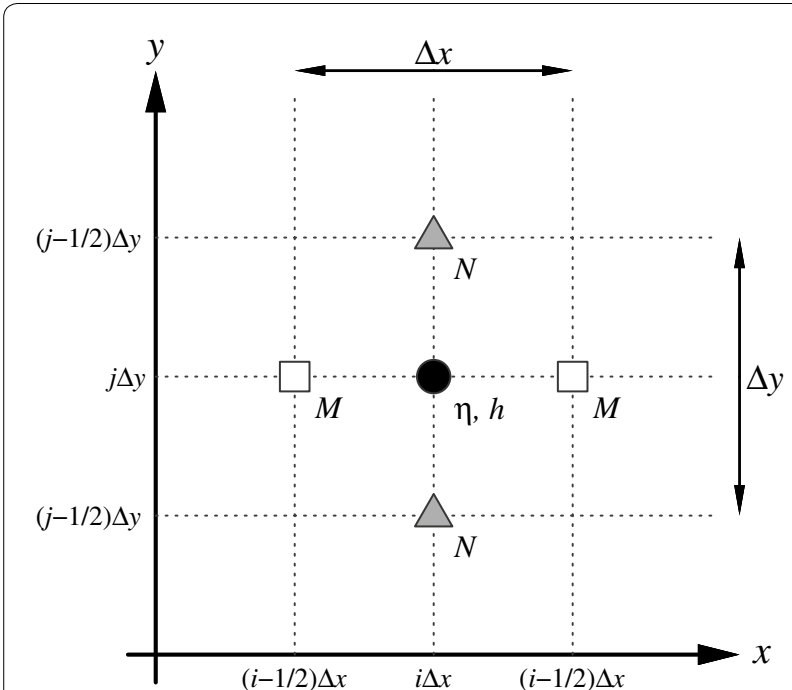

Fig. 1 Staggered-grid layout adopted in this study for tsunami simulation by the finite difference method

\section{Linear dispersive-wave tsunami equation}

For the LDW equations, the momentum equation becomes numerically implicit in time due to the existence of the dispersion term. To solve this linear system, we adopted an iterative procedure based on Saito et al. (2010) but with a slight modification. For computational efficiency, we first solved the derivatives with respect to time of the tsunami velocity components, $\dot{M}(x, y, t)$ and $\dot{N}(x, y, t)$, rather than calculating the tsunami velocity by implicitly solving Eq. 10, where the dots denote derivatives with respect to time. Integration with respect to time was then explicitly performed by using the estimated derivatives with respect to time of the tsunami velocities $\dot{M}(x, y, t)$ and $\dot{N}(x, y, t)$. The detailed discretized equations are given in Appendix.

In the PML region, the spatial derivatives of the dispersion term were easily calculated by using the definition of the dispersion term, Eq. 11, as follows: 


$$
\begin{aligned}
& \left(\partial_{x} \Phi\right)_{i-1 / 2, j}^{n}=\dot{M}_{i-1 / 2, j}^{n}+g \bar{h}_{i-1 / 2, j} \frac{\eta_{i, j}^{n}-\eta_{i-1, j}^{n}}{\Delta x} \\
& \left(\partial_{y} \Phi\right)_{i, j-1 / 2}^{n}=\dot{N}_{i, j-1 / 2}^{n}+g \bar{h}_{i, j-1 / 2} \frac{\eta_{i, j}^{n}-\eta_{i, j-1}^{n}}{\Delta y} .
\end{aligned}
$$

The tsunami velocities, $M$ and $N$, at the $(n+1 / 2)$-th time step were calculated as follows:

$$
\begin{aligned}
M_{i-1 / 2, j}^{n+1 / 2}= & \frac{\beta_{(x) i-1 / 2}-\delta_{(x) i-1 / 2} \Delta t / 2}{\beta_{(x) i-1 / 2}+\delta_{(x) i-1 / 2} \Delta t / 2} M_{i-1 / 2, j}^{n-1 / 2} \\
& +\frac{\Delta t}{\beta_{(x) i-1 / 2}+\delta_{(x) i-1 / 2} \Delta t / 2} \\
& \left(-g \bar{h}_{i-1 / 2, j} \frac{\eta_{i, j}^{n}-\eta_{i-1, j}^{n}}{\Delta x}+w_{i-1 / 2}\left(\partial_{x} \Phi\right)_{i-1 / 2, j}^{n}\right) \\
N_{i, j-1 / 2}^{n+1 / 2}= & \frac{\beta_{(y) j-1 / 2}-\delta_{(y) j-1 / 2} \Delta t / 2}{\beta_{(y) j-1 / 2}+\delta_{(y) j-1 / 2} \Delta t / 2} N_{i, j-1 / 2}^{n-1 / 2} \\
& +\frac{\Delta t}{\beta_{(y) j-1 / 2}+\delta_{(y) j-1 / 2} \Delta t / 2} \\
& \left(-g \bar{h}_{i, j-1 / 2} \frac{\eta_{i, j}^{n}-\eta_{i, j-1}^{n}}{\Delta y}+w_{j-1 / 2}\left(\partial_{y} \Phi\right)_{i, j-1 / 2}^{n}\right) .
\end{aligned}
$$

Substituting Eq. 17 in Eq. 18, we obtained the discretized momentum equations for tsunami velocity in the PML region as follows:

$$
\begin{aligned}
M_{i-1 / 2, j}^{n+1 / 2}= & \frac{\beta_{(x) i-1 / 2}-\delta_{(x) i-1 / 2} \Delta t / 2}{\beta_{(x) i-1 / 2}+\delta_{(x) i-1 / 2} \Delta t / 2} M_{i-1 / 2, j}^{n-1 / 2} \\
& +\frac{\Delta t}{\beta_{(x) i-1 / 2}+\delta_{(x) i-1 / 2} \Delta t / 2} \\
& \left(\left(w_{i-1 / 2}-1\right) g \bar{h}_{i-1 / 2, j} \frac{\eta_{i, j}^{n}-\eta_{i-1, j}^{n}}{\Delta x}+w_{i-1 / 2} \dot{M}_{i-1 / 2, j}^{n}\right) \\
N_{i, j-1 / 2}^{n+1 / 2}= & \frac{\beta_{(y) j-1 / 2}-\delta_{(y) j-1 / 2} \Delta t / 2}{\beta_{(y) j-1 / 2}+\delta_{(y) j-1 / 2} \Delta t / 2} N_{i, j-1 / 2}^{n-1 / 2} \\
& +\frac{\Delta t}{\beta_{(y) j-1 / 2}+\delta_{(y) j-1 / 2} \Delta t / 2} \\
& \left(\left(w_{j-1 / 2}-1\right) g \bar{h}_{i, j-1 / 2} \frac{\eta_{i, j}^{n}-\eta_{i, j-1}^{n}}{\Delta y}+w_{j-1 / 2} \dot{N}_{i, j-1 / 2}^{n}\right) .
\end{aligned}
$$

In the final form, Eq. 19, the dispersion term does not explicitly appear. We note that Eq. 19 asymptotes to a simple numerical integration (Eq. 23) when $\beta_{(*)} \rightarrow 1$, $\delta_{(*)} \rightarrow 0$, and $w \rightarrow 1$.

\section{Numerical examples}

\section{Smooth bathymetry model}

To examine the effectiveness of the proposed PML absorbing boundary conditions for numerical simulation of a tsunami, we performed a finite difference simulation for a smoothly sloping bathymetric structure as depicted in Fig. 2. In this smooth bathymetry model, the assumed ocean depths are typical of the bathymetry from a continental shelf to a trench associated with a subduction zone at a plate boundary. The simulation model was discretized with a grid width of $\Delta x=\Delta y=500 \mathrm{~m}$ and a time step of $\Delta t=1.0 \mathrm{~s}$.

To assess the effect of artificial reflections from the model boundary, we compared simulation results in the smaller bounded region (region 1 in Fig. 2) with the results for a reflection-free model, which was derived from a larger model (region 2 in Fig. 2) as a reference. We compared the simulated tsunami for the following three types of absorbing boundary conditions: (1) a one-way boundary condition, (2) a sponge boundary condition, and (3) the PML boundary condition proposed in this report. All of these boundary conditions were applied on the outer boundary of region 1 (Fig. 2).

The one-way boundary condition uses the one-way wave equation, with the wave propagating perpendicular to the boundary at the outermost grid (e.g., Tanioka and Seno 2001). The sponge boundary uses the scheme of Cerjan et al. (1985) with an absorber thickness of 20 grids. We used the same thickness for the PML region.

We set the initial sea-surface height at time $t=0$ as the tsunami source. We used the following two-dimensional cosine function (Hossen et al. 2015b) for the initial seasurface height:

$$
\begin{gathered}
\eta(x, y, t=0)=\frac{\eta_{0}}{4}\left[1+\cos \left(\frac{\pi\left(x-x_{0}\right)}{A_{x}}\right)\right]\left[1+\cos \left(\frac{\pi\left(y-y_{0}\right)}{A_{y}}\right)\right] \\
\text { for }\left|x-x_{0}\right| \leq A_{x},\left|y-y_{0}\right| \leq A_{y},
\end{gathered}
$$

where $A_{x}$ and $A_{y}$ are characteristic source sizes. We set $A_{x}=A_{y}=16 \mathrm{~km}$. The maximum initial height at $x=x_{0}$ and $y=y_{0}$ was assumed to be $\eta_{0}=1 \mathrm{~m}$. The source locations $x_{0}$ and $y_{0}$ were equated to the center of the model.

Figure 3 and Additional file 1 show snapshots of the tsunami wavefield based on the LLW model simulation for the three types of absorbing boundary conditions as well as the reflection-free reference model. The snapshots show the tsunami waves spreading isotropically from the initial height given by Eq. 20. At the second time step of the snapshot $(t=800 \mathrm{~s})$, the tsunami wavefront reaches the boundaries of region 1 at $y=0 \mathrm{~km}$ (bottom) and at $x=0$ and $200 \mathrm{~km}$. It is apparent that the one-way and sponge boundary conditions cause artificial reflections from the boundaries. The reflections are much stronger for the one-way boundary than for the sponge boundary. As time passes, these artificially reflected waves propagate back to the model interior. At $t=1300 \mathrm{~s}$, the entire simulation area (region 1) is contaminated by the 


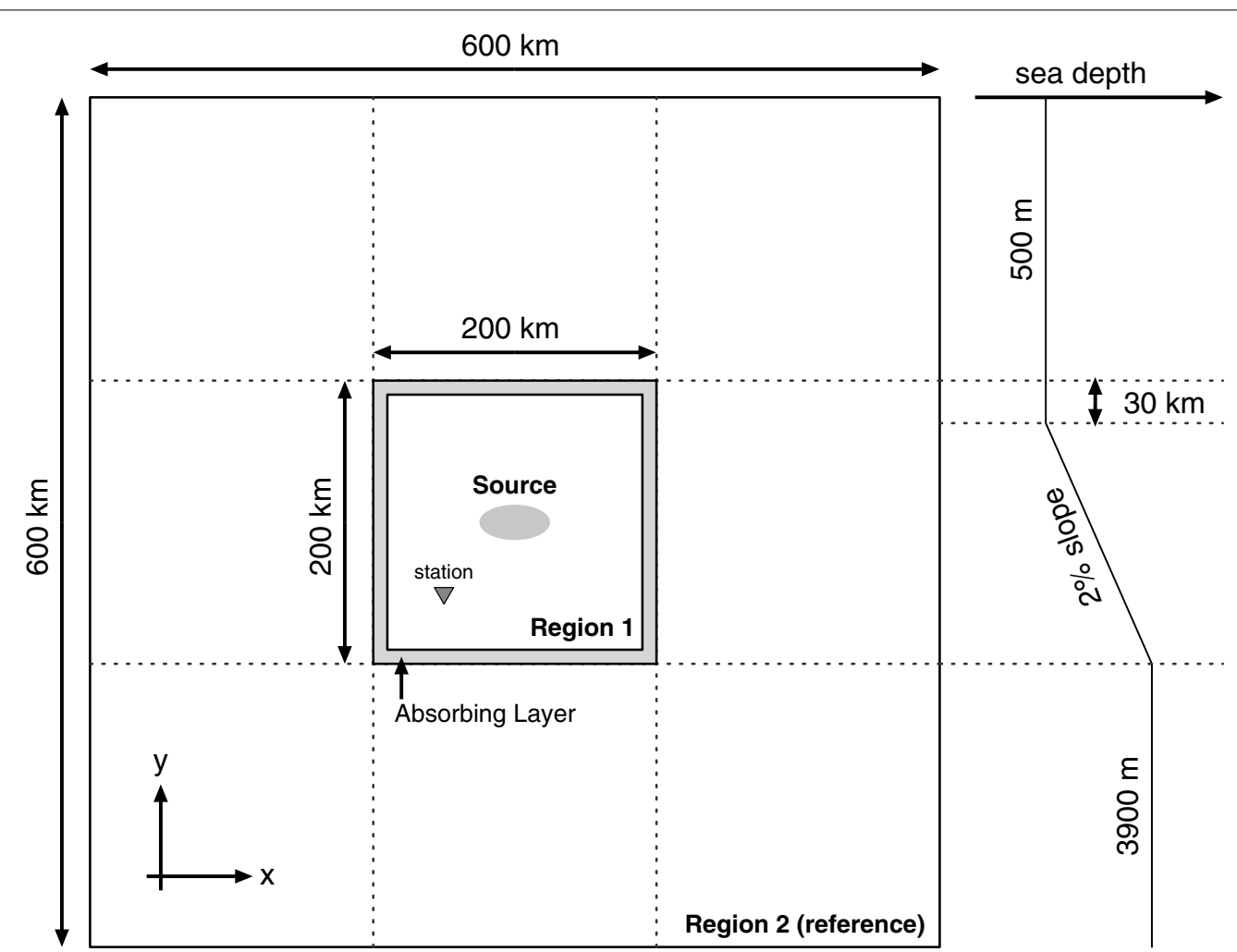

Fig. 2 A schematic illustration of a bathymetric model used to test the effects of different boundary conditions on tsunami wave propagation

artificial reflections from the boundaries. In contrast, the PML absorbing condition generates very weak, almost negligible, reflections from the boundaries compared to the one-way and sponge boundary conditions.

The results in Fig. 4 and Additional file 2 demonstrate the similar effectiveness of the PML boundary conditions for the LDW model. Because the characteristic size of the assumed source area is not as large $\left(A_{x}=A_{y}=16 \mathrm{~km}\right)$ as the ocean depth $(3.9 \mathrm{~km}$ at the deepest point), a dispersive tsunami wave train was very clearly apparent, especially in the $y$-direction. As a result, dispersion considerably elongated the tsunami wave packet by 800 and $1300 \mathrm{~s}$. The results of the tsunami simulation using the one-way and sponge boundary conditions show that the dispersed later phases (800 and 1300 s) were completely overlapped by the artificial reflections from the boundary at $y=0 \mathrm{~km}$. In particular, the reflection for the one-way boundary condition was much stronger than in the case of the LLW model, probably because of the mismatch between the dispersed tsunami velocity and the assumed tsunami velocity used in the one-way wave equation (Fig. 3a). In contrast, the PML boundary condition very efficiently suppressed such artificial reflections. At a very late elapsed time of $t=1300 \mathrm{~s}$, the amplitude of the reflected wave was only on the order of $1 \mathrm{~cm}$, which is much smaller than the amplitude of reflected waves for the one-way and sponge boundary conditions.

The one-way boundary condition assumed that the tsunami wave propagated perpendicular to the boundary with the speed of a linear long-wave. However, dispersion effects cause the long-wave condition to be inconsistent with the LDW tsunami model. The difference between the tsunami propagation speeds derived from the longwave assumption is probably the cause of the significant artificial reflection. The sponge condition generally attenuates a tsunami at all speeds; however, snapshots (Figs. 3, 4) made it apparent that the sponge boundary condition caused significant reflections when the approaching wave was nearly parallel to the boundary. That the PML boundary condition effectively attenuated only the components propagating outward from the boundary resulted in the best reduction of reflections. The effectiveness of the PML boundary condition can also be confirmed by comparing simulated tsunami traces (Fig. 5). For the tsunami waveforms obtained in simulations based on the one-way and sponge boundary conditions, the artificial reflections from the boundary observed at times near $t=600-1400 \mathrm{~s}$ for both the LLW and LDW models were much larger than those obtained with the reference model. On the contrary, the reflected waves 


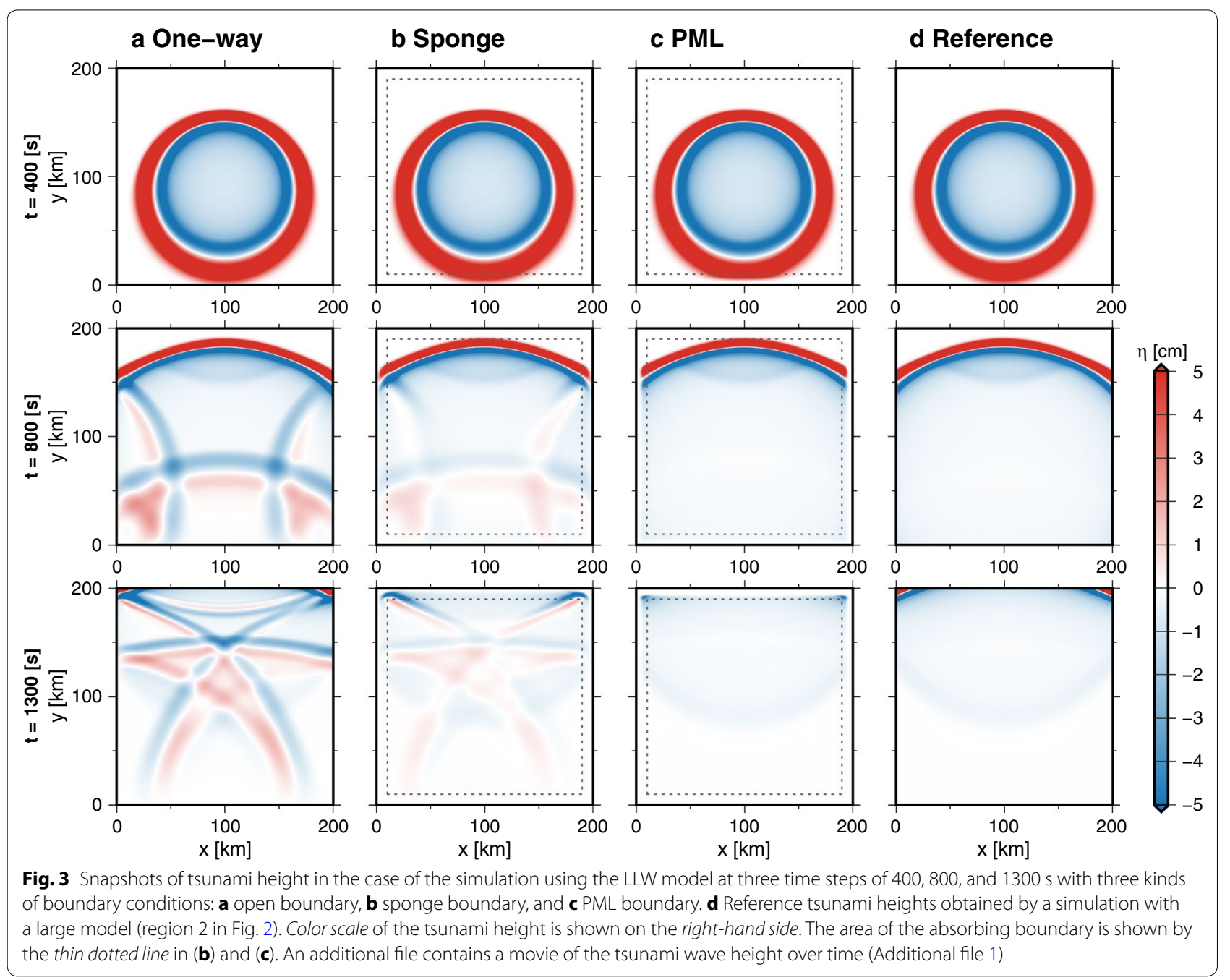

were almost indistinguishable for the simulation using the PML boundary condition. The artificial reflections can be easily identified in the case of the LLW model, because they are temporally separated from the arrival of the main tsunami signal. However, in the case of the more realistic tsunami simulation with the LDW model, the dispersed later tsunami arrivals were significantly contaminated by artificial reflections from boundary. It is thus rather difficult to distinguish the contamination of artificial reflections in the tsunami waveforms. The PML boundary condition was more effective in reducing the risk of artificial reflections in the LDW tsunami simulation model.

Tsunami simulation in Nankai Trough in southwest Japan We next applied the PML boundary condition to simulation of a tsunami wave propagating in the Nankai Trough, southwest of Japan (Fig. 6). The model had a spatial extent of $1050 \mathrm{~km}$ (east-west) $\times 550 \mathrm{~km}$ (north-south), which was almost adequate to cover the Nankai Trough southwest of Japan, where the Philippine Sea plate subducts beneath the Japanese archipelago. The model was discretized in space and time with grid widths of $\Delta x=\Delta y=1000 \mathrm{~m}$ and $\Delta t=1.0 \mathrm{~s}$.

The initial sea-surface height was a single circular elevation of the sea surface based on Eq. 20 with $A_{x}=A_{y}=22.5 \mathrm{~km}$ and was centered near the Kii peninsula. This simulation mimicked the problem of calculating the Green's function for purposes of tsunami forecasting and/or inversion of the initial sea-surface height of large earthquakes (e.g., Tsushima et al. 2009; Baba and Cummins 2005; Takagawa and Tomita 2014).

To demonstrate the effectiveness of the PML absorbing boundary conditions, we compared the simulated tsunami wavefield with the wavefield in the reflection-free reference model. We extended the simulation area of the reference model $500 \mathrm{~km}$ southward to avoid boundaryreflected waves. We note that waves reflected from the 

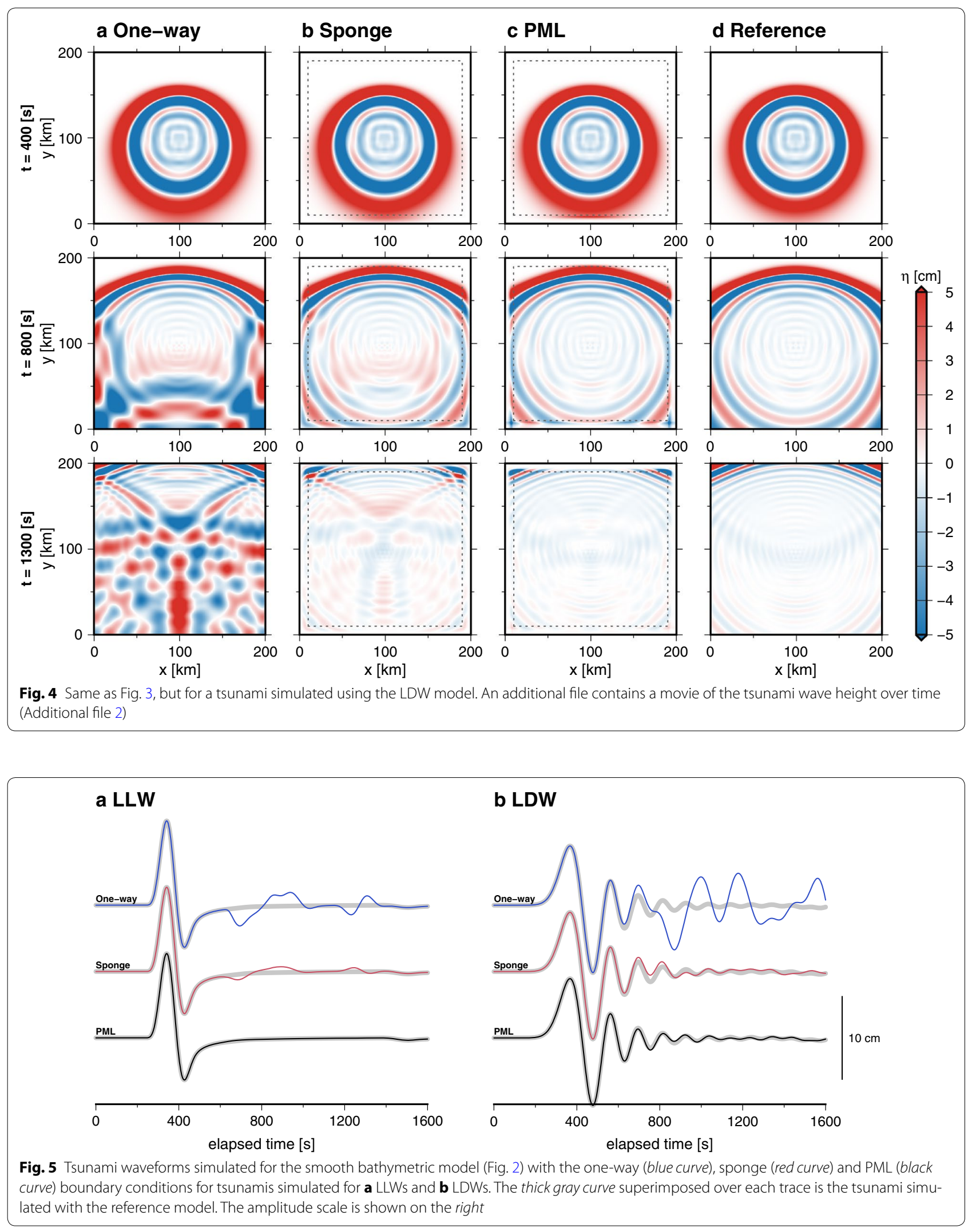


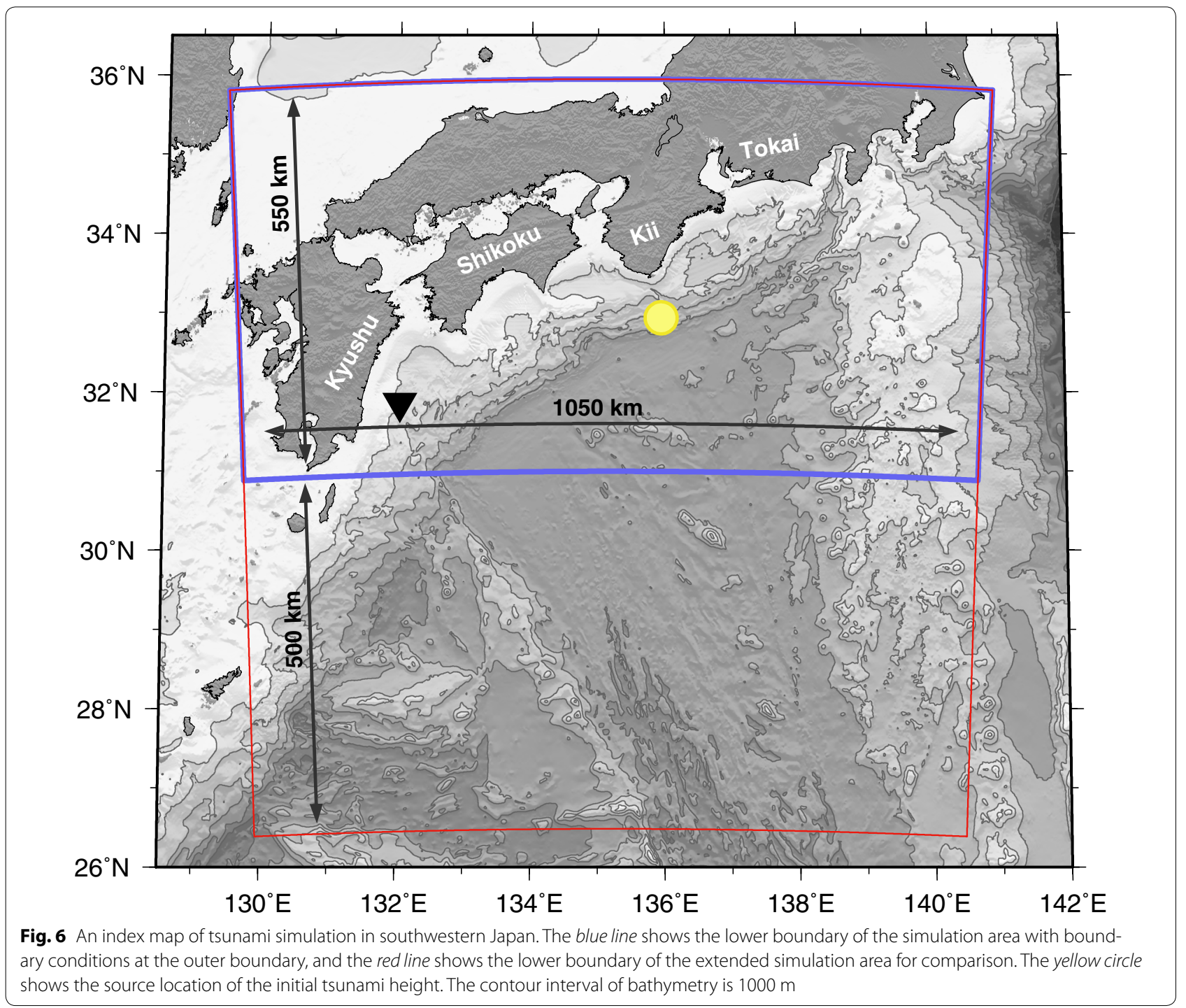

boundaries on the other side of the model area did not occur until the end of the simulation because the tsunami wave propagation speed toward these boundaries was slowed by the shallow bathymetry.

Snapshots of the propagation of this tsunami by the LLW model are shown in Fig. 7 and Additional file 3. We also show the residual tsunami wavefield obtained by subtracting the wavefield of the reference model (Fig. 7) to emphasize the presence of the artificial reflection from the southern boundary. Because of the faster tsunami propagation speed, the reflected wave from the southern boundary quickly approached the Japanese archipelago. The amplitude increased as the tsunami speed decreased in the cases of the one-way and sponge boundaries. The reflection was especially strong at Shikoku and Kyushu islands in the west because the boundary was closer. At an elapsed time of $2500 \mathrm{~s}$, the wavefront of the reflected wave was approaching the direct wavefront near Kyushu.

In contrast, there was no significant reflection in the case of the model with a PML boundary. Some of the signal in the snapshot of the residual amplitude at an elapsed time of $2500 \mathrm{~s}$ in the PML boundary model was backscattering of the tsunami in the reference model area (red rectangle in Fig. 6) that originated from outside the model area (blue rectangle in Fig. 6) rather than an artificial reflection from the model boundary. We note, however, that such backscattering does not affect tsunami waves near the coast of Japan, because the origin of the scattering is far from the southern boundary.

In the case of tsunami simulation with the LDW model, the reflection from the southern boundary was much worse (Fig. 8 and Additional file 4), especially for the 

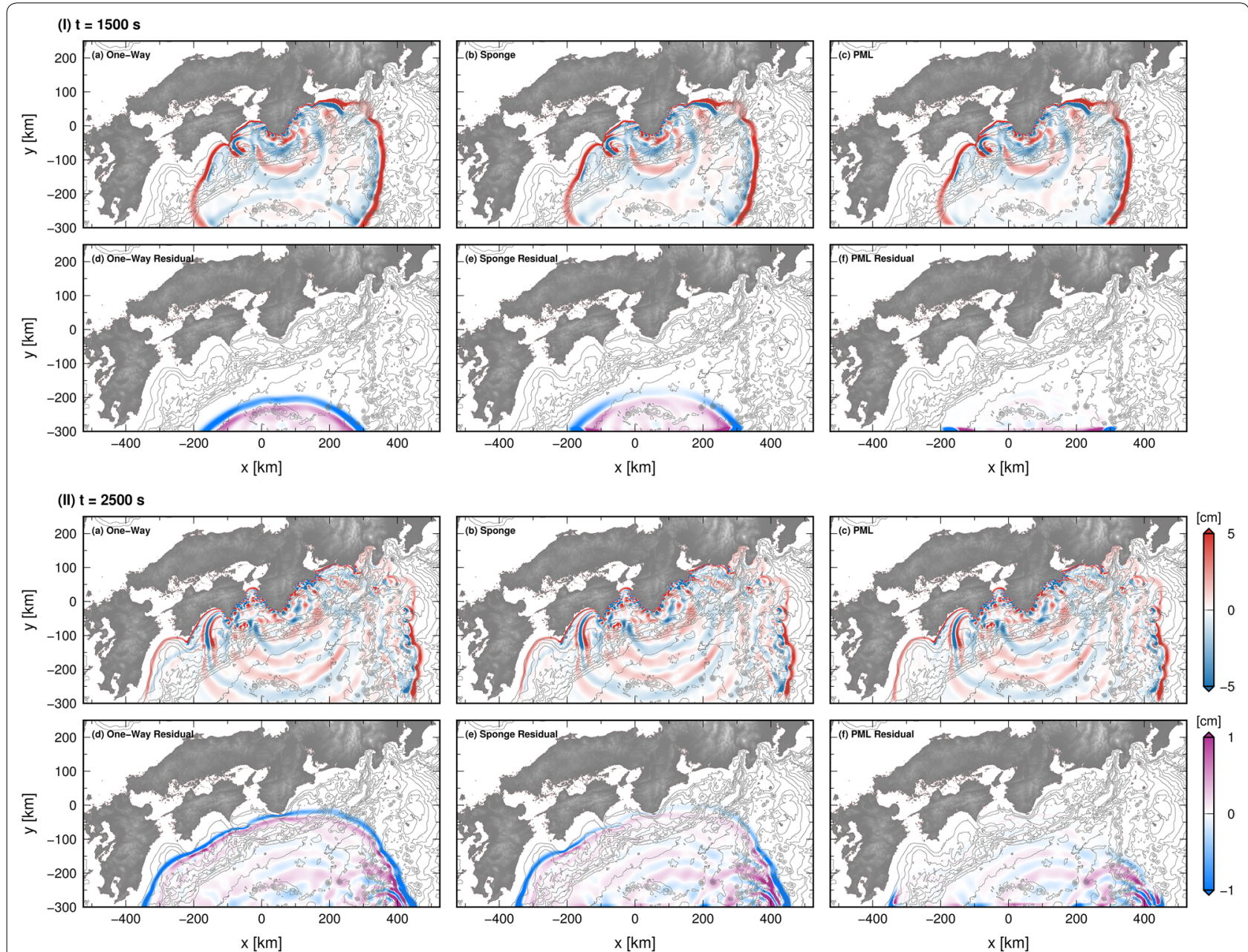

Fig. 7 Snapshots of the simulation of tsunami propagation for the LLW model at two time steps of $\mathbf{I} t=1500 \mathrm{~s}$ and $\mathbf{I I} \mathrm{t}=2500 \mathrm{~s}$ using $a$ one-way, $b$ sponge, and c PML boundary conditions. In each time step, top panels $a-c$ show tsunami height for each simulation. The bottom panels $d-f$ show the residual tsunami heights obtained by subtracting from the tsunami heights the heights of the reference numerical simulation over a wider region (see Fig. 6). Color scales are shown on the right hand side. An additional file contains a movie of the tsunami wave height over time (Additional file 3)

one-way boundary condition, as was the case for the simple bathymetry model. The reflected wave spread widely because of dispersion effects. The sponge boundary condition gave better results than the one-way boundary condition; however, there were still large artifacts, particularly at wide angles of reflection. In contrast, the PML boundary conditions gave excellent results, even in the LDW model, with respect to elimination of artificial reflections. We note again that a major part of the residual tsunami wavefield was the scattered waves from outside the simulation model, and they did not penetrate near the trough axis.

Figure 9 shows the comparison of tsunami waveforms recorded at an ocean-bottom station (see Fig. 6 for location) near Kyushu Island. On the one hand, the tsunami waveforms of the LLW models with one-way and sponge boundary conditions showed a large tsunami packet with reversed polarity, i.e., a reflected wave. On the other hand, no visible reflection appeared in the simulation with PML boundary conditions. The contamination of the complicated reflected waves were generated in the LDW model (Fig. 9b) because of the dispersion of both the direct and boundary-reflected waves. The use of the PML boundary conditions allowed us to use the later phases of the tsunami and will thus facilitate more detailed estimations of tsunami sources and/or forecasts of tsunamis.

\section{Discussion and conclusions}

We developed and demonstrated the use of an effective absorbing boundary condition for finite difference simulation of tsunamis. The new PML boundary condition for 


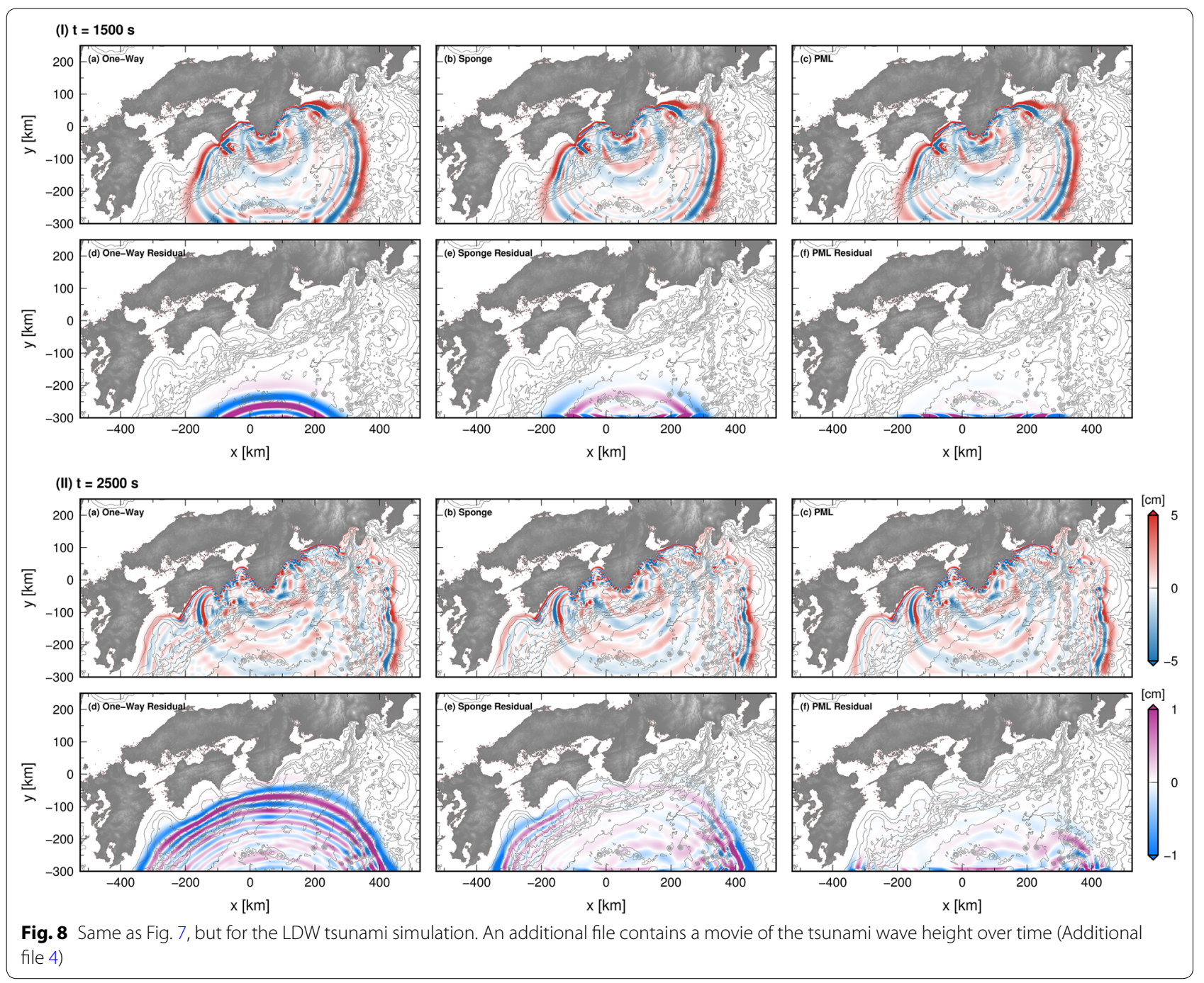

LLW tsunamis was empirically extended to LDW tsunamis and was shown to be very effective in reducing artificial reflections of tsunamis at the model boundaries. The effectiveness of the PML boundary condition may be sensitive to the PML damping parameters, but we confirmed that it is rather robust and a set of same PML parameters previously used in the modeling of seismic waves (Zhang and Shen 2010) work efficiently for tsunami waves as well.

Although the model was developed using Cartesian coordinates, it is straightforward to extend the model to polar coordinates. However, it may be necessary to neglect the effect of the Coriolis force, at least in the PML region. Because the effect of the Coriolis force is expected to be quite small for regional tsunami modeling (Inazu and Saito 2015), ignoring the Coriolis force will not be an obstacle to achieving efficient absorbing conditions.
The wave speed of a tsunami depends on the depth of the ocean, and large variations of depth may enhance the effect of artificial reflections. A tsunami usually propagates very rapidly offshore $(\sim 300 \mathrm{~m} / \mathrm{s}$ at a depth of $10,000 \mathrm{~m})$ and slowly around a coastline $(\sim 50 \mathrm{~m} / \mathrm{s}$ at a depth $300 \mathrm{~m}$ ). As a result, artificial reflections at the open boundary in a deep ocean return to the model interior at a very high speed, as shown in Figs. 3 and 4. Because the amplitude of a tsunami generally increases with decreasing depth of the water column, such artificial reflections are significantly enhanced around coastlines. This effect may have a strong influence on the use of tsunami waveforms at later phases of the tsunami, e.g., in the case of a dispersive tsunami wave.

In practice, such artificial reflections can be avoided by simply extending the model area, and such an extension is not very difficult for the LLW model. However, 


\section{a LLW}

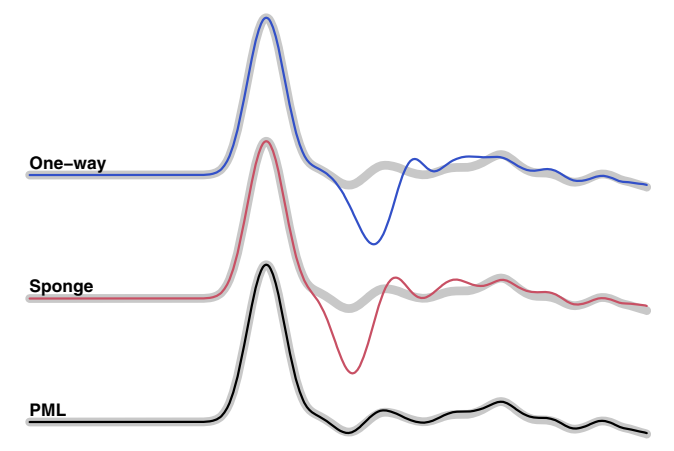

1600

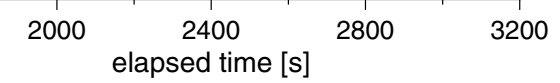

b LDW

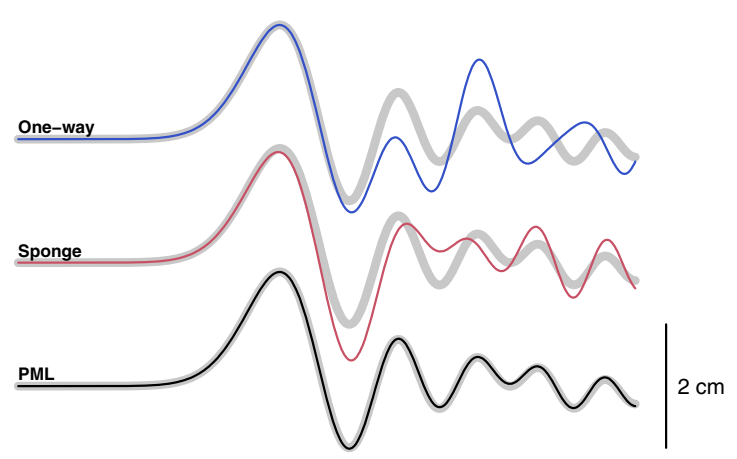

1600
2400

elapsed time [s]

Fig. 9 Same as Fig. 5, but for the model of southwestern Japan (Fig. 6)

simulation of a tsunami with the LDW model is usually very expensive in terms of computational costs, which are roughly 30-100 times the costs of LLW tsunami simulations because of the iterative processes required to solve the finite difference equations. We note that because the PML condition is applied only once, during the last of the iterations (see Eq. 19), the additional cost of introducing the PML is negligibly small. Therefore, use of the PML absorbing boundary condition alternative to extending the model area greatly reduces computational costs. The rapid, high-resolution simulation of tsunamis with robust absorbing boundary conditions for a model of moderate size should be useful for the repetitive simulation of tsunamis that is required, for example, for inversion studies of source fault ruptures and/or computation of the large number of Green's functions (e.g., Baba and Cummins 2005; Tsushima et al. 2009) required for tsunami forecasting or source inversion.

\section{Availability and requirements}

Project name: tsunami_pml

Project home page: http://github.com/takuto-maeda/ tsunami_pml

Operating system: Linux and OS X

Programing Language: Fortran2008

Other requirements: None

License: MIT license

Any restrictions to use by non-academics: None

\section{Additional files}

Additional file 1. An time-sequential animation file asociated in Fig. 3. Additional file 2. An time-sequential animation file asociated in Fig. 4. Additional file 3. An time-sequential animation file asociated in Fig. 7. Additional file 4. An time-sequential animation file asociated in Fig. 8.

\section{Abbreviations}

PML: perfectly matched layer; LLW: linear long-wave; LDW: linear dispersive-wave.

\section{Authors' contributions}

TM developed the PML theory for tsunami simulation and the method of implementation for numerical integration. TM and HT participated in developing the simulation codes and carrying out the numerical tests. TF participated in the conception and design of important intellectual components of the report. All authors contributed to drafting the manuscript. All authors read and approved the final manuscript.

\section{Author details}

${ }^{1}$ Earthquake Research Institute, The University of Tokyo, 1-1-1 Yayoi, Bunkyo-ku, Tokyo 113-0032, Japan. ${ }^{2}$ Meteorological Research Institute, Japan Meteorological Agency, 1-1 Nagamine, Tsukuba-shi, Ibaraki 305-0052, Japan.

\section{Acknowledgements}

The JTOPO30v2 bathymetry model data that were used in this study were provided by the Marine Information Research Center, Japan Hydrographic Association. We thank Kentaro Imai and Daisuke Inazu for their valuable suggestions. Constructive comments and suggestions from Hiroshi Takenaka, the editor, and an anonymous reviewer are greatly appreciated. TM was supported by the Japan Society for the Promotion of Science (JSPS) KAKENHI Grant number $15 \mathrm{~K} 16306$.

\section{Competing interests}

The authors declare that they have no competing interests. 


\section{Appendix: Discretization of dispersive tsunami \\ equation}

Equation 10 was discretized in space with the following second-order finite-difference scheme:

$$
\begin{aligned}
M_{i-1 / 2, j}^{n+1 / 2} & =M_{i-1 / 2, j}^{n-1 / 2}+\dot{M}_{i-1 / 2, j}^{n} \Delta t \\
N_{i, j-1 / 2}^{n+1 / 2} & =N_{i, j-1 / 2}^{n-1 / 2}+\dot{N}_{i, j-1 / 2}^{n} \Delta t
\end{aligned}
$$

$$
\begin{aligned}
\dot{M}_{i-1 / 2, j}^{n}= & -g h_{i-1 / 2, j}\left(\frac{\eta_{i, j}^{n}-\eta_{i-1, j}^{n}}{\Delta x}\right)+\frac{h_{i-1 / 2, j}^{2}}{3} \frac{\dot{M}_{i+1 / 2, j}^{n}-2 \dot{M}_{i-1 / 2, j}^{n}+\dot{M}_{i-3 / 2, j}^{n}}{\Delta x^{2}} \\
& +\frac{h_{i-1 / 2, j}^{2}}{3} \frac{\dot{N}_{i, j+1 / 2}^{n}-\dot{N}_{i, j-1 / 2}^{n}-\dot{N}_{i-1, j+1 / 2}^{n}+\dot{N}_{i-1, j-1 / 2}^{n}}{\Delta x \Delta y} \\
\dot{N}_{i, j-1 / 2}^{n}= & -g h_{i, j-1 / 2}\left(\frac{\eta_{i, j}^{n}-\eta_{i, j-1}^{n}}{\Delta y}\right)+\frac{h_{i, j-1 / 2}^{2}}{3} \frac{\dot{N}_{i, j+1 / 2}^{n}-2 \dot{N}_{i, j-1 / 2}^{n}+\dot{N}_{i, j-3 / 2}^{n}}{\Delta y^{2}} \\
& +\frac{h_{i, j-1 / 2}^{2}}{3} \frac{\dot{M}_{i+1 / 2, j}^{n}-\dot{M}_{i+1 / 2, j-1}^{n}-\dot{M}_{i-1 / 2, j}^{n}+\dot{M}_{i-1 / 2, j-1}^{n}}{\Delta x \Delta y} .
\end{aligned}
$$

We used the Gauss-Seidel method to solve Eq. 21. The derivative with respect to time of the tsunami velocity was updated as follows based on Eq. 21 and an implicit scheme:

$$
\begin{aligned}
\dot{M}_{i-1 / 2, j}^{n}= & -\frac{3 \Delta x^{2}}{3 \Delta x^{2}+2 h_{i-1 / 2, j}^{2}} g h_{i-1 / 2, j}\left[\frac{\eta_{i, j}^{n}-\eta_{i-1, j}^{n}}{\Delta x}\right] \\
& +\frac{h_{i-1 / 2, j}^{2}}{3 \Delta x^{2}+2 h_{i-1 / 2, j}^{2}}\left[\dot{M}_{i+1 / 2, j}^{n}+\dot{M}_{i-3 / 2, j}^{n}\right] \\
& +\frac{h_{i-1 / 2, j}^{2}}{3 \Delta x^{2}+2 h_{i-1 / 2, j}^{2}} \frac{\Delta x}{\Delta y}\left[\dot{N}_{i, j+1 / 2}^{n}-\dot{N}_{i, j-1 / 2}^{n}-\dot{N}_{i-1, j+1 / 2}^{n}+\dot{N}_{i-1, j-1 / 2}^{n}\right] \\
\dot{N}_{i, j-1 / 2}^{n}= & -\frac{3 \Delta y^{2}}{3 \Delta y^{2}+2 h_{i, j-1 / 2}^{2}} g h_{i, j-1 / 2}\left[\frac{\eta_{i, j}^{n-1 / 2}-\eta_{i, j-1}^{n-1 / 2}}{\Delta y}\right] \\
& +\frac{h_{i, j-1 / 2}^{2}}{3 \Delta y^{2}+2 h_{i, j-1 / 2}^{2}}\left[\dot{N}_{i, j+1 / 2}^{n}+\dot{N}_{i, j-3 / 2}^{n}\right] \\
& +\frac{h_{i, j-1 / 2}^{2}}{3 \Delta y^{2}+2 h_{i, j-1 / 2}^{2}} \frac{\Delta y}{\Delta x}\left[\dot{M}_{i+1 / 2, j}^{n}-\dot{M}_{i+1 / 2, j-1}^{n}-\dot{M}_{i-1 / 2, j}^{n}+\dot{M}_{i-1 / 2, j-1}^{n}\right]
\end{aligned}
$$

Equation 22 was repeatedly applied until $\dot{M}_{i-1 / 2, j}^{n}$ and $\dot{N}_{i, j-1 / 2}^{n}$ converged. Tsunami velocities in the interior region were then updated by numerical integration with respect to time as follows: 
Received: 29 December 2015 Accepted: 4 April 2016

Published online: 23 April 2016

\section{References}

Baba T, Cummins PR (2005) Contiguous rupture areas of two Nankai Trough earthquakes revealed by high-resolution tsunami waveform inversion. Geophys Res Lett 32:L08305. doi:10.1029/2004GL022320

Baba T, Takahashi N, Kaneda Y, Ando K, Matsuoka D, Kato T (2015) Parallel implementation of dispersive tsunami wave modeling with a nesting algorithm for the 2011 Tohoku Tsunami. Pure appl Geophys 172:34553472. doi:10.1007/s00024-015-1049-2

Berenger J-P (1994) A perfectly matched layer for the absorption of electromagnetic waves. J Comput Phys 114(2):185-200. doi:10.1006/ jcph.1994.1159

Blumberg AF, Kantha LH (1985) Open boundary condition for circulation models. J Hydraul Eng 111:237-255. doi:10.1061/ (ASCE)0733-9429(1985)111:2(237)

Cerjan C, Kosloff D, Kosloff R, Reshef M (1985) A nonreflecting boundary condition for discrete acoustic and elastic wave equations. Geophysics 50:705-708. doi:10.1190/1.441945

Chew WC, Liu QH (1996) Perfectly matched layers for elastodynamics: a new absorbing boundary condition. J Comput Acoust 4:341-359. doi:10.1142/ S0218396X96000118

Festa G, Nielsen S (2003) PML absorbing boundaries. Bull Seism Soc Am 93(2):891-903. doi:10.1785/0120020098

Furumura T, Saito T (2009) Integrated ground motion and tsunami simulation for the 1944 Tonankai earthquake using high-performance supercomputers. J Disaster Res 4:118-126

Furumura T, Imai K, Maeda T (2011) A revised tsunami source model for the 1707 Hoei earthquake and simulation of tsunami inundation of Ryujin Lake, Kyushu, Japan. J Geophys Res 116:B02308. doi:10.1029/201 OJB007918

González FI, Kulikov YA (1993) Tsunami dispersion observed in the deep ocean. In: Tinti S (ed) Tsunamis in the World. Kluwer Acad, Dordrecht, pp 7-16. doi:10.1007/978-94-017-3620-6_2

Goto C (1984) Equation of nonlinear dispersive long waves for a large Ursell number. J Hydraul Coast Environ Eng 351:193-201 (in Japanese with English abstract)

Grilli ST, Harris JC, Bakhsh TST, Masterlark TL, Kyriakopoulos C, Kirby JT, Shi F (2013) Numerical simulation of the 2011 Tohoku tsunami based on a new transient FEM co-seismic source: comparison to far- and nearfield observations. Pure appl Geophys 170:1333-1359. doi:10.1007/ s00024-012-0528-y

Hossen MJ, Cummins PR, Dettmer J, Baba T (2015a) Tsunami waveform inversion for sea surface displacement following the 2011 Tohoku earthquake: importance of dispersion and source kinematics. J Geophys Res Solid Earth. doi:10.1002/2015JB011942

Hossen MJ, Cummins PR, Dettmer J, Baba T (2015b) Time reverse imaging for far-field tsunami forecasting: 2011 Tohoku earthquake case study. Geophys Res Lett. doi:10.1002/2015GL065868

Hwang L-S, Butler HL, Divoky DJ (1972) Tsunami model: generation and opensea characteristics. Bull Seism Soc Am 62:1579-1596

Inazu D, Saito T (2015) Global tsunami simulation using a grid rotation transformation in a latitude-longitude coordinate system. Nat Hazards. doi:10.1007/s11069-015-1995-0

Kim KY, Reid RO, Whitaker RE (1988) On an open radiational boundary condition for weakly dispersive tsunami waves. J Comput Phys 76:327-348. doi:10.1016/0021-9991(88)90146-5

Lavelle JW, Thacker WC (2008) A pretty good sponge: dealing with open boundaries in limited-area ocean models. Ocean Model 20:270-292. doi:10.1016/j.ocemod.2007.10.002

Maeda T, Furumura T (2013) FDM simulation of seismic waves, ocean acoustic waves, and tsunamis based on the seismic-tsunami compound equations. Pure appl Geophys 170:109-127. doi:10.1007/s00024-011-0430-z
Marcinkovich C, Olsen K (2003) On the implementation of perfectly matched layers in a three-dimensional fourth-order velocity-stress finite difference scheme. J Geophys Res 108(B5):2276. doi:10.1029/2002JB002235

Moczo P, Robertsson JOA, Eisner $L$ (2007) The finite-difference method for modeling of seismic wave propagation. Adv Geophys 48:421-516. doi:10.1016/S0065-2687(06)48008-0

Navon IM, Neta B, Hussaini MY (2004) A perfectly matched layer approach to the linearized shallow water equations models. Mon Weather Rev 132:1369-1378. doi:10.1175/1520-0493(2004)132<1369:APMLAT>2.0. $\mathrm{CO} ; 2$

Press WH, Teukolsky SA, Vetterling WT, Flannery BP (1986) Numerical recipes. Cambridge University Press, Cambridge

Saito T, Furumura T (2009) Three-dimensional simulation of tsunami generation and propagation: application to intraplate events. J Geophys Res 114:B02307. doi:10.1029/2007JB005523

Saito T, Satake K, Furumura T (2010) Tsunami waveform inversion including dispersive waves: the 2004 earthquake off Kii Peninsula, Japan. J Geophys Res 115:B06303. doi:10.1029/2009JB006884

Saito T, Ito Y, Inazu D, Hino R (2011) Tsunami source of the 2011 Tohoku-Oki earthquake, Japan: inversion analysis based on dispersive tsunami simulations. Geophys Res Lett 38:L00G19. doi:10.1029/2011GL049089

Takagawa T, Tomita T (2014) Effects of rupture processes in an inverse analysis on the tsunami source of the 2011 Off the Pacific coast of Tohoku earthquake. Int J Offshore Polar Eng 24(1):21-27

Tanioka Y (1999) Analysis of the far-field tsunamis generated by the 1998 Papua New Guinea earthquake. Geophys Res Lett 26(22):3393-3396. doi: 10.1029/1999GL005392

Tanioka Y (2006) Rupture process of the 2004 great Sumatra-Andaman earthquake estimated from tsunami waveforms. Earth Planets Space 58:203-209. doi:10.1186/BF03353379

Tanioka Y, Seno T (2001) Detailed analysis of tsunami waveforms generated by the 1946 Aleutian tsunami earthquake. Nat Hazards Earth Syst Sci 1:171-175. doi:10.5194/nhess-1-171-2001

Tsushima H, Hino R, Fujimoto H, Tanioka Y, Imamura F (2009) Near-field tsunami forecasting from cabled ocean bottom pressure data. J Geophys Res 114:B06309. doi:10.1029/2008JB005988

Zhang W, Shen Y (2010) Unsplit complex frequency-shifted PML implementation using auxiliary differential equations for seismic wave modeling. Geophysics 75(4):T141-T154. doi:10.1190/1.3463431

\section{Submit your manuscript to a SpringerOpen ${ }^{\odot}$ journal and benefit from:}

- Convenient online submission

- Rigorous peer review

- Immediate publication on acceptance

- Open access: articles freely available online

- High visibility within the field

- Retaining the copyright to your article

Submit your next manuscript at springeropen.com 